\title{
ON AN INVERSION FORMULA FOR THE LAPLACE TRANSFORMATION, II
}

\author{
P. G. ROONEY
}

In an earlier paper (3) we discussed at some length a certain inversion operator for the Laplace transformation. If

$$
\text { I } \quad f(s)=\int_{0}^{\infty} e^{-s t} \phi(t) d t=\mathscr{L}(\phi(t) ; s)
$$

then the inversion operator is given by

$$
\text { II } \quad{ }^{\nu} L_{k, t}[f(s)]=\frac{k^{3 / 2} e^{2 k}}{t \pi^{\frac{1}{3}}} \int_{0}^{\infty} x^{\frac{1}{2} \nu} J_{\nu}\left(2 k x^{\frac{3}{3}}\right) f\left(\frac{k(x+1)}{t}\right) d x,
$$

and we showed that if $\nu>-1$, then under certain conditions

$$
\lim _{k \rightarrow \infty}{ }^{\nu} L_{k, t}[f(s)]=\phi(t) .
$$

It is our purpose here to discuss the behaviour of the operator for $\nu \leqslant-1$.

It is clear that if $\nu \leqslant-1$ and $\nu$ is not an integer, the operator will not exist. For, by $(2, \S 7.2 .1,(2))$,

$$
x^{\frac{1}{2} \nu} J_{\nu}\left(2 k x^{\frac{1}{2}}\right)=(k x)^{\nu}(1+o(1)) / \Gamma(\nu+1), \quad x \rightarrow 0+,
$$

if $\nu$ is not a negative integer. However, if $\nu$ is a negative integer, $\nu=-n$, a different situation appears, for, by $(2, \S 7.2 .4,(24)), J_{-n}(z)=(-1)^{n} J_{n}(z)$, and hence

$$
x^{-\frac{1}{2} n} J_{-n}\left(2 k x^{\frac{1}{2}}\right)=(-1)^{n} k^{n}(1+o(1)) / n ! \quad x \rightarrow 0+.
$$

Thus there is some prospect of the operator existing in this case.

It will transpire that the operator will exist if $\nu=-n$, under certain hypotheses on $\phi(t)$, and that a suitable modification of the operator will invert the transformation. The theory is contained in the following two theorems.

We make use of the notation

$$
\int_{0}^{\rightarrow \infty} \phi(u) d u
$$

to denote the 'Improper' Lebesgue integral. That is,

$$
\int_{0}^{\rightarrow \infty} \phi(u) d u=\lim _{R \rightarrow \infty} \int_{0}^{R} \phi(u) d u .
$$

Also we define,

$$
\text { III } \quad{ }^{\nu} \overrightarrow{L_{k, t}}[f(s)]=\frac{k^{3 / 2} e^{2 k}}{t \pi^{\frac{1}{3}}} \int_{0}^{\rightarrow \infty} x^{\frac{1}{2} \nu} J_{\nu}\left(2 k x^{\frac{1}{2}}\right) f\left(\frac{k(x+1)}{t}\right) d x \text {. }
$$

Received February 7, 1955. This paper is based on work done at the Summer Research Institute of the Canadian Mathematical Congress. 
ThEOREM 1. If $e^{-\gamma t} \phi(t) \in L(0, \infty), \gamma>0$, then

$$
{ }^{-n} \overrightarrow{L_{k}, t}[f(s)]
$$

exists for each $t>0$ and all $k>\gamma t,(n=1,2, \ldots)$, and

$$
{ }^{-n} \vec{L}_{k, t}[f(s)]=(-t)^{-n}{ }^{0} L_{k, t}\left[f^{(n)}(s)\right]-\frac{(-1)^{n-1} k^{n+\frac{1}{2}} e^{2 k}}{\pi^{\frac{1}{2}}} \sum_{r=1}^{n} \frac{(k t)^{-r}}{(n-r) !} f^{(r-1)}\left(\frac{k}{t}\right) .
$$

Proof. By (1; ch. $3, \S 2), f^{(n)}(s)=L\left((-t)^{n} \phi(t) ; s\right), s>\gamma$, and thus, by (3, Theorem 2.1), ${ }^{0} L_{k, t}\left[f^{(n)}(s)\right]$ exists for $k>\gamma t(n=1,2, \ldots)$.

Let $k>\gamma t$. Then, since

$$
\frac{d}{d z} J_{0}(z)=-J_{1}(z)=J_{-1}(z)
$$

and since $J_{0}(z)$ and $f(z) \rightarrow 0$ as $z \rightarrow \infty$, we obtain on integrating by parts,

$$
\begin{aligned}
& { }^{0} L_{k, t}\left[f^{\prime}(s)\right]=\frac{k^{3 / 2} e^{2 k}}{t \pi^{\frac{1}{3}}} \int_{0}^{\infty} J_{0}\left(2 k x^{\frac{1}{2}}\right) f^{\prime}\left(\frac{k(x+1)}{t}\right) d x \\
& =\frac{k^{3 / 2} e^{2 k}}{t \pi^{\frac{3}{3}}}\left\{\left.\frac{t}{k} J_{0}\left(2 k x^{\frac{1}{2}}\right) f\left(\frac{k(x+1)}{t}\right)\right|_{0} ^{\infty}-t \int_{0}^{\infty} x^{-\frac{1}{2}} J_{-1}\left(2 k x^{\frac{1}{2}}\right) f\left(\frac{k(x+1)}{t}\right) d x\right\} \\
& =-\frac{k^{\frac{1}{2}} e^{2 k}}{\pi^{\frac{3}{3}}} f\left(\frac{k}{t}\right)-t \cdot{ }^{-1}{\overrightarrow{L_{k}}}_{t}[f(s)],
\end{aligned}
$$

which is the stated result for $n=1$.

We now proceed by induction. Assuming the result true for $n$, we have, since $f^{\prime}(s)=\mathscr{L}(-t \phi(t) ; s)$ for $s>\gamma$, that

$$
{ }^{-n} \overrightarrow{L_{k, t}}\left[f^{\prime}(s)\right]
$$

exists for $k>\gamma t$ and equals

$$
(-t)^{-n 0} L_{k, t}\left[f^{(n+1)}(s)\right]-\frac{(-1)^{n-1} k^{n+\frac{1}{2}}}{\pi^{\frac{1}{2}}} \sum_{r=1}^{n} \frac{(k t)^{-r}}{(n-r) !} f^{(r)}\left(\frac{k}{t}\right) .
$$

Then, for $k>\gamma t$, since, by $(2, \S 7.2 .8,(51))$,

$$
\frac{d}{d z} z^{-n} J_{-n}(z)=z^{-n} J_{-(n+1)}(z)
$$

and $J_{-n}(z)$ and $f(z) \rightarrow 0$ as $z \rightarrow \infty$, we have, on integration by parts, that

$$
\begin{aligned}
& { }^{-n}{\overrightarrow{L_{k, t}}}_{f}\left[f^{\prime}(s)\right]=\frac{k^{3 / 2} e^{2 k}}{t \pi^{\frac{1}{2}}} \int_{0}^{\rightarrow \infty} x^{-\frac{1}{2} n} J_{-n}\left(2 k x^{\frac{1}{2}}\right) f^{\prime}\left(\frac{k(x+1)}{t}\right) d x \\
& =\frac{k^{3 / 2} e^{2 k}}{t \pi^{\frac{3}{3}}}\left\{\left.\frac{t}{k} x^{-\frac{1}{2} n} J_{-n}\left(2 k x^{\frac{1}{2}}\right) f\left(\frac{k(x+1)}{t}\right)\right|_{0} ^{\infty}-t \int_{0}^{\rightarrow \infty} x^{-\frac{1}{2}(n+1)} J_{-(n+1)}\left(2 k x^{\frac{1}{2}}\right)\right. \\
& =-\frac{(-1)^{n} k^{n+\frac{1}{2}} e^{2 k}}{\pi^{\frac{1}{3}} n !}-t \cdot{ }^{-(n+1)} \vec{L}_{k, t}[f(s)] \\
& f\left(\frac{k(x+1)}{t}\right) d x
\end{aligned}
$$


Hence,

$$
\begin{aligned}
& { }^{-(n+1)} \vec{L}_{k, t}[f(s)]=-t^{-1} \cdot{ }^{-n} \overrightarrow{L_{k}, t}\left[f^{\prime}(s)\right]-\frac{(-1)^{n} k^{n+\frac{1}{2}} e^{2 k}}{t \pi^{\frac{1}{3}} n !} f\left(\frac{k}{t}\right) \\
= & (-t)^{-(n+1)}{ }^{0} L_{k, t}\left[f^{(n+1)}(s)\right]+\frac{(-1)^{n-1} k^{n+\frac{1}{2}} e^{2 k}}{t \pi^{\frac{1}{3}}} \sum_{r=1}^{n} \frac{(k t)^{-r}}{(n-r) !} f^{(r)}\left(\frac{k}{t}\right) \\
= & \quad-\frac{(-1)^{n} k^{n+\frac{1}{2}} e^{2 k}}{t \pi^{\frac{1}{2}} n !} f\left(\frac{k}{t}\right) \\
& (-t)^{-(n+1)}{ }^{0} L_{k, t}\left[f^{(n+1)}(s)\right]-\frac{(-1)^{n} k^{n+3 / 2} e^{2 k}}{\pi^{\frac{1}{2}}} \sum_{r=1}^{n+1} \frac{(k t)^{-r}}{(n+1-r) !} f^{(r-1)}\left(\frac{k}{t}\right) .
\end{aligned}
$$

Hence the formula is true for all $n$.

Corollary. If $e^{-\gamma t} \phi(t) \in L(0, \infty), \gamma>0$, and if $t^{-\frac{1}{6}} \phi(t) \in L(0, \delta)$, then ${ }^{-1} L_{k, t}[f(s)]$ exists and

$$
{ }^{-1} L_{k, t}[f(s)]=-t^{-1} L_{k, t}\left[f^{\prime}(s)\right]-\frac{k^{\frac{1}{2}} e^{2 k}}{\pi^{\frac{1}{2}} t} f\left(\frac{k}{t}\right) .
$$

If $e^{-\gamma t} \phi(t) \in L(0, \infty), \gamma>0$, then ${ }^{-n} L_{k, t}[f(s)]$ exists for $n=2,3, \ldots$, and

$$
{ }^{-n} L_{k, t}[f(s)]=(-t)^{-n 0} L_{k, t}\left[f^{(n)}(s)\right]-\frac{(-1)^{n-1} k^{n+\frac{1}{2}} e^{2 k}}{\pi^{\frac{1}{2}}} \sum_{r=1}^{n} \frac{(k t)^{-r}}{(n-r) !} f^{(r-1)}\left(\frac{k}{t}\right) .
$$

Proof. The existence of ${ }^{-n} L_{k, t}[f(s)]$ under the various hypotheses follows exactly as in (3, Theorem 2.1$)$. The stated relations now follow from Theorem 1 , since

$$
{ }^{-n} \vec{L}_{k, t}[f(s)]={ }^{-n} L_{k, t}[f(s)]
$$

when both exist.

Theorem 2. If $e^{-\gamma t} \phi(t) \in L(0, \infty), \gamma>0$, then at each point $t>0$ of the Lebesgue set of $\phi$,

$$
\lim _{k \rightarrow \infty}\left\{-n \vec{L}_{k, t}[f(s)]+\frac{(-1)^{n-1} k^{n+\frac{1}{2}} e^{2 k}}{\pi^{\frac{1}{2}}} \sum_{r=1}^{n} \frac{(k t)^{-r}}{(n-r) !} f^{(r-1)}\left(\frac{k}{t}\right)\right\}=\phi(t) .
$$

Proof. This now follows from Theorem 1, and Theorem 3.1 of (3).

Corollary. If $e^{-\gamma t} \phi(t) \in L(0, \infty), \gamma>0$, and if $t^{-\frac{1}{4}} \phi(t) \in L(0, \delta)$, for some $\delta>0$, then at each point $t>0$ of the Lebesgue set of $\phi$,

$$
\lim _{k \rightarrow \infty}\left\{{ }^{-1} L_{k, t}[f(s)]+\frac{k^{3 / 2} e^{2 k}}{\pi^{\frac{1}{3}} t k} f\left(\frac{k}{t}\right)\right\}=\phi(t) .
$$

If $e^{-\gamma t} \phi(t) \in L(0, \infty), \gamma>0$, then at each point $t>0$ of the Lebesgue set of $\phi$,

$$
\lim _{k \rightarrow \infty}\left\{{ }^{-n} L_{k, t}[f(s)]+\frac{(-1)^{n-1} k^{n+\frac{1}{2}} e^{2 k}}{\pi^{\frac{3}{2}}} \sum_{r=1}^{n} \frac{(k t)^{-r}}{(n-r) !} f^{(r-1)}\left(\frac{k}{t}\right)\right\}=\phi(t)
$$

for $n=2,3, \ldots$. 
Proof. This now follows from the corollary to Theorem 1, and Theorem 3.1 of (3).

\section{REFERENCES}

1. G. Doetsch, Handbuch der Laplace-Transformation, vol. 1 (Basel, 1950).

2. A. Erdélyi, Higher transcendental functions (Bateman Manuscript Project, Vol. 2, New York, 1954).

3. P. G. Rooney, On an inversion formula for the Laplace transformation, Can. J. Math., $\tilde{}$ (1955), 101-115.

University of Toronto 